\title{
Relação do comportamento do perfil do estilo de vida de escolares com o de seus pais
}

\author{
The relationship between the behavior of students' lifestyle profile \\ and that of their parents
}

Tatiane Minuzzi (https://orcid.org/0000-0003-2558-6709) ${ }^{1}$

Kelly Christine Maccarini Pandolfo (https://orcid.org/0000-0002-6215-9768) ${ }^{2}$

Cati Reckelberg Azambuja (https://orcid.org/0000-0003-3836-3264) ${ }^{1}$

Daniela Lopes dos Santos (https://orcid.org/0000-0002-1782-1337) ${ }^{1}$
${ }^{1}$ Centro de Educação Física e Desporto, Universidade Federal de Santa Maria. Av. Roraima 1000, Camobi. 97105-900 Santa Maria RS Brasil.

tatiane.minuzzi@gmail.com ${ }^{2}$ Colégio Militar de Santa Maria. Santa Maria RS Brasil.

\begin{abstract}
There is evidence that parental support and behavior impact the healthy behavior of adolescents directly. The scope of this study is to identify the relationship between students' Lifestyle Profile (LP) and that of their parents. Two hundred and eight students aged between 11 and 15 and their respective parents participated in the study. An instrument that included five components was used to evaluate the parents' LP. These components included nutrition, physical activity, preventive behavior, relationships, and stress management. An instrument adapted for adolescents was used to evaluate the students' LP. Descriptive statistics and the Chi-square test were used for the data analysis. Students, mothers and fathers showed positive behavior (PB) for the $L P$, but when the different components of lifestyle were stratified, an average score of the nutritional responses of students and parents was bordering on negative behavior (NB). The average number of mothers' points in physical activity corresponded to NB. There were no significant relationships between parents' and students' LP, though in the physical activity component alone a significant relationship was found. The relationships established between the groups in the LP suggest that $P B$ of the parents increases the chances of having children with $P B$.
\end{abstract}

Key words Lifestyle, Parents, Children
Resumo Há evidências que o apoio e o comportamento parental interferem diretamente no comportamento saudável dos adolescentes. O objetivo deste estudo é identificar a relação do Perfil de Estilo de Vida (PEV) parental com os estudantes. Participaram 208 escolares, de 11 a 15 anos, e seus respectivos pais (mãe/pai). Para avaliar o PEV dos pais foi utilizado um instrumento que engloba cinco componentes associados ao estilo de vida: nutrição, atividade física, comportamento preventivo, relacionamentos e controle do estresse. Já, para os alunos foi aplicado o instrumento adaptado para adolescentes. A análise dos resultados foi através da estatística descritiva e o teste Qui-Quadrado. Os alunos, as mães e os pais apresentaram um comportamento positivo (CP) para os componentes PEV, a pontuação média das respostas na nutrição dos alunos e dos pais ficou próxima a um comportamento negativo (CN). A média de pontos das mães na atividade física correspondeu a um CN. Não houve relações significativas dos componentes do PEV do grupo casais com o grupo de filhos, somente no componente atividade fisica foi encontrada uma relação significativa. As relações estabelecidas entre os grupos nos componentes sugerem que uma postura conjunta positiva de ambos os pais possibilita aumentar a possibilidade de ter filhos com $C P$.

Palavras-chave Estilo de vida, Pais, Filhos 


\section{Introdução}

A adolescência é um período da vida no qual ocorrem intensas mudanças físicas e psicológicas e que nesta fase, podem ser determinados os hábitos que serão levados para a vida adulta, evidencias têm apontado que o apoio e comportamento parental interferem diretamente no desenvolvimento e manutenção do comportamento saudável adotado pelos adolescentes ${ }^{1-3}$. Tal fase é favorável para intervenções e modificações de hábitos e comportamentos, pois é um período caracterizado por processos de crescimento e desenvolvimento, em que ocorrem diversas mudanças biopsicossociais de forma concomitante e marcante. Todas estas modificações tornam os adolescentes mais vulneráveis a fatores de riscos à saúde e a mudanças no modo de viver, com interferência direta na qualidade de vida ${ }^{4}$.

A Qualidade de vida $(\mathrm{QV})$ é reconhecida como a percepção do indivíduo de sua posição na vida, um conceito que representa as respostas individuais aos efeitos físicos, mentais e sociais da doença sobre o cotidiano, e que influencia a extensão em que a satisfação pessoal com as circunstâncias da vida pode ser adquirida ${ }^{5}$. Dentre os comportamentos de risco à saúde que compõem a QV e que devem ser urgentemente modificados pelos jovens, destacam-se os relacionados ao estilo de vida $(\mathrm{EV})^{6}$. Nas últimas décadas vivenciou-se uma impactante alteração nos padrões do EV da população em geral. Diversos fatores como o processo de urbanização, aparecimento de doenças e, principalmente, o desenvolvimento industrial e das tecnologias vêm causando transformações nos diversos segmentos da rotina, repercutindo na qualidade de vida dos indivíduos?.

$\mathrm{O} \mathrm{EV}$, que é definido como o conjunto de ações habituais e modificáveis que refletem as atitudes, os valores e as oportunidades na vida das pessoas, passou a ser um dos mais importantes determinantes da saúde dos indivíduos, grupos e comunidades ${ }^{8}$. De acordo com Gonçalves e Vilarta ${ }^{9}$, estilo de vida é caracterizado por hábitos que os indivíduos aprendem e adotam durante suas vidas, que se relacionam com a realidade familiar, ambiental e social. Os autores reforçam que durante a vida o ser humano se apropria de diversos conjuntos de hábitos que envolvem a biologia e a cultura e estes fatores podem interferir nos seus aspectos de saúde e bem-estar.

Para Carvalho e Mineiro ${ }^{10}$ o conceito de estilo de vida tem ganho muita importância no campo de intervenção da educação para a saúde e isto se deve ao fato de que a solução para os problemas atuais no que se refere à saúde, dependem mais do que nunca, das opções que os sujeitos tomam no dia-a-dia, seja ele ao nível físico, mental, social ou espiritual, sugerindo que a manutenção da saúde depende grandemente do comportamento adotado pelo sujeito.

Nahas ${ }^{8}$ determinou que o EV é constituído de um conjunto de cinco componentes: nutrição, atividade física, comportamento preventivo, relacionamento social e controle do estresse. Tudo indica que estes são passíveis de modificação, por meio de interferências positivas e que podem ser controlados pelas pessoas em seu dia a dia. Por outro lado, adotar um EV pouco saudável, quando você cria um perfil negativo, ou seja, quando assume hábitos como a inatividade física, a ingestão de alimentos em exagero, e principalmente gordurosos, fumo e alcoolismo, aliados a uma vida cheia de stress contribui para o aparecimento de várias doenças e aumento do risco da mortalidade ${ }^{8}$.

Os aspectos relacionados ao EV de adolescentes ainda são pouco investigados no Brasil, especialmente no que diz respeito a influência do comportamento de risco à saúde dos seus pais. Pressupõe-se que os pais possam influenciar os filhos na adoção de comportamentos relacionados à saúde, pois a família constitui o primeiro ambiente de aprendizagem e referência das crianças e adolescentes ${ }^{11}$. Neste sentindo, objetivou-se verificar a associação do PEV dos pais com o PEV dos filhos (alunos) do Ensino Fundamental de uma escola pública federal do sul do Brasil.

\section{Métodos}

Este trabalho foi desenvolvido por meio de uma pesquisa de corte transversal descritivo correlacional. Foram convidados a participar 1.275 sujeitos entre escolares (425) matriculados no ano de 2016 no Ensino Fundamental ( $6^{\circ}$ ao $9^{\circ}$ ano), e seus respectivos pais (425) e mães (425). Com as recusas e o não cumprimento dos critérios de inclusão, participaram efetivamente da pesquisa o total de 624 sujeitos, sendo: 208 escolares do ensino fundamental (116 meninas e 92 meninos) e seus respectivos pais (208) e mães (208). Como critérios de inclusão, adotaram-se: apresentar o Termo de Consentimento e Assentimento Livre Esclarecido (TCALE) assinado pelos pais ou responsáveis, e também pelo próprio aluno, alem de responder os instrumentos da pesquisa, de forma completa, dentro do prazo estabelecido. 
Após contato com a Direção da escola, a fim de solicitar a autorização para a realização do estudo em suas dependências, fez-se o contato com os sujeitos da pesquisa para a informação dos objetivos do estudo, esclarecimentos acerca da coleta de dados e solicitação de suas participações através do TCALE. O trabalho foi conduzido dentro dos padrões éticos exigidos pela Declaração de Helsinque de 1964 e de acordo com a resolução 466/12 do Ministério da Saúde. O estudo foi aprovado pelo Comitê de Ética em Pesquisa (CEP) da Universidade Federal de Santa Maria (UFSM).

No levantamento das informações referentes aos comportamentos do estilo de vida relacionado à saúde dos pais, aplicou-se um questionário denominado Perfil do Estilo de Vida - PEV (índice do bem-estar $)^{12}$. Para avaliar o PEV dos alunos foi utilizado o mesmo questionário, adaptado para adolescente, que segue o mesmo modelo de avaliação do questionário do pais porém com as perguntas adaptadas para a faixa etária ${ }^{8}$.

Trata-se de um modelo de avaliação que engloba cinco fatores ou componentes associados ao estilo de vida de indivíduos ou grupos: nutrição, atividade física, comportamento preventivo, relacionamentos e controle do estresse. O questionário possui 15 itens de perguntas fechadas, os chamados hábitos de estilo de vida. As respostas correspondem a uma auto avaliação do tipo escalar, em que 0 (zero) não faz parte do seu estilo de vida, 1 (um) às vezes corresponde ao comportamento, 2 (dois) quase sempre verdadeiro e 3 (três) faz parte do seu estilo de vida. Para o cálculo do estilo de vida, foram considerados os escores médios de cada componentes do PEV. Um estilo de vida negativo, ou seja, aquele que oferece riscos à saúde e afeta a qualidade de vida, foi classificado dentro do intervalo de média de pontos entre 0 e 1,5 e o positivo de 1,6 e 3 pontos $^{8}$.

A coleta de dados foi realizada em julho de 2016, durante as aulas de Educação Física realizado em um auditório com todos os alunos de cada ano, onde responderam os questionários individualmente. Já os questionários destinados aos pais, foram enviados através dos próprios alunos, dentro de um envelope lacrado, contendo os questionários para ambos os pais e as explicações necessárias para o preenchimento adequado. Foi estabelecida uma semana para o retorno dos mesmos.

Foi realizada análise descritiva das médias das respostas para caracterizar a grupo estudado. Para verificar as relações entre o resultado do comportamento dos escolares nos componentes do PEV com o resultados do comportamento do grupo dos pais nos componentes do PEV foi realizado Teste Qui-Quadrado $(\mathrm{p}<0,5)$.

\section{Resultados}

Do total de 1.275 sujeitos convidados a participar da pesquisa, somente 624 se enquadraram nos critérios de inclusão (escolares $=208$, pais $=$ 208 e mães $=208$ ), sendo $55,77 \%$ alunos do sexo feminino $(\mathrm{n}=116)$ e $44,23 \%$ do sexo masculino ( $\mathrm{n}=92$ ), com idade média de 12,95 $\pm 1,44$ anos, variando a idade de 11 à 15 anos. Os pais apresentaram idade média de 44,5 $\pm 5,85$ anos e as mães uma idade média de 42,08 $\pm 5,17$ anos.

A média das respostas dos escolares, das mães e dos pais apresentaram de forma geral um comportamento positivo para o PEV. Porém pode se observar que a média das respostas da nutrição dos alunos e dos pais foi próximo a um comportamento negativo $(\mathrm{CN})(1,56 \pm 0,60$ pontos $)$. A média das respostas das mães na atividade física $(1,20 \pm 0,84$ pontos) demostrou que as mesmas possuem um comportamento negativo $(\mathrm{CN})$, e próximo ao um $\mathrm{CN}$ no controle de estresse $(1,60$ $\pm 0,71$ pontos) (Tabela 1 ).

Para a realização da análise de correlação entre o comportamento dos pais (casal) e filhos nos componentes do $\mathrm{PEV}$, os pais foram agrupados da seguinte maneira: casal concordam com comportamento positivo $\left(\mathrm{C}^{+}\right)$, quando ambos possuem comportamento positivo para o $\mathrm{PEV}$; casal concordam comportamento negativo $\left(\mathrm{C}^{-}\right)$, quando ambos possuem comportamento negativo para o PEV; casal discorda pai comportamento positivo $\left(\mathrm{P}^{+}\right)$, quando o pai possui comportamento positivo e mãe comportamento negativo no casal; casal discorda mãe com comportamento positivo $\left(\mathrm{M}^{+}\right)$, quando o pai possui comportamento negativo e a mãe tem comportamento positivo.

Verifica-se na Tabela 2 que não houve relação dos comportamentos entre filhos e pais no componente nutrição do PEV ( $p>0,5)$. Apesar disso, é possível notar numericamente que quando ambos os pais possuem $\mathrm{CP}\left(\mathrm{C}^{+}\right), 60,5 \%$ deles têm filhos com CP e 39,5\% têm filhos com CN. Quando ambos possuem $\mathrm{CN}\left(\mathrm{C}^{-}\right)$, 60,5\% deles têm filhos com $\mathrm{CN}$. Isto parece indicar que, quando ambos os pais possuem CP, há uma maior chance de terem filhos também com CP na nutrição, assim como quando ambos possuem $\mathrm{CN}$ parece aumentar a possibilidade de os filhos terem um CN. Porém, quando apenas um deles apresenta 
$\mathrm{CN}$, isso parece não comprometer o comportamento nutricional do filho.

Ao analisar as relações dos comportamentos entre pais e filhos no componente AF, a Tabela 3 demonstra que o comportamento do grupo de pais (casal) está relacionado ao comportamento do grupo de filhos $(p=0,015)$. Quando ambos os pais possuem $\mathrm{CP}\left(\mathrm{C}^{+}\right)$no componente $\mathrm{AF}, 95,6 \%$ deles têm filhos com CP e 4,4\% dos pais com filhos com $\mathrm{CN}$. Entretanto, quando ambos possuem $\mathrm{CN}\left(\mathrm{C}^{-}\right), 73,1 \%$ deles têm filhos com CP. Isto indica que, quando ambos os pais possuem $\mathrm{CP}$, há maior tendência de ter filhos também com CP. Porém, quando ambos os pais ou apenas um deles apresentam $\mathrm{CN}$, isso parece não prejudicar o comportamento positivo do filho na AF. Cabe ressaltar que, em pequena magnitude, quando ambos os pais possuem um $\mathrm{CN}$, parece crescer o número de filhos com $\mathrm{CN}(51 \%)$, quando comparado ao comportamento desigual entre o casal.

Ao se analisar as relações dos comportamentos entre pais e filhos nos componentes comportamento preventivo, relacionamento e controle do estresse do PEV, nas Tabelas 4, 5 e 6 respectivamente, verifica-se que o comportamento do grupo de pais (casal) não esteve relacionado ao comportamento do grupo de filhos. Porém, numericamente, pode-se observar quando ambos os pais possuem comportamento positivo $\left(\mathrm{C}^{+}\right)$: há um maior percentual de filhos com comportamento positivo, quando comparado nos outros grupos de casais, podendo-se inferir que há uma

Tabela 1. Médias das respostas do questionário Perfil do Estilo de Vida (PEV) em cada componente.

\begin{tabular}{lcccccc}
\hline \multicolumn{1}{c}{$\begin{array}{c}\text { Grupo de Estudo } \\
\text { Variáveis (em pontos) }\end{array}$} & \multicolumn{2}{c}{ Filhos } & \multicolumn{2}{c}{ Mães } & \multicolumn{2}{c}{ Pais } \\
\cline { 2 - 7 } & $\mathbf{X}$ & DP & X & DP & X & DP \\
\hline Nutrição & 1,56 & 0,60 & 1,73 & 0,49 & 1,56 & 0,60 \\
Atividade física & 1,99 & 0,49 & $1,20^{*}$ & 0,84 & 1,58 & 0,77 \\
Comportamento preventivo & 2,72 & 0,42 & 2,60 & 0,48 & 2,54 & 0,49 \\
Relacionamento Social & 2,25 & 0,54 & 2,08 & 0,68 & 2,05 & 0,70 \\
Controle de estresse & 2,17 & 0,62 & 1,60 & 0,71 & 1,84 & 0,68 \\
\hline
\end{tabular}

Legenda: X: média; DP: desvio padrão; "Hábitos classificados como negativos.

Tabela 2. Análise da relação do componente nutrição do PEV entre pais e filhos.

\begin{tabular}{|c|c|c|c|c|c|c|c|c|c|c|c|c|c|c|}
\hline \multirow{3}{*}{ PEV-N } & \multicolumn{6}{|c|}{$\begin{array}{c}\text { Casal Concorda } \\
\text { (comportamento) }\end{array}$} & \multicolumn{7}{|c|}{$\begin{array}{c}\text { Casal Discorda } \\
\text { (comportamento) }\end{array}$} & \multirow{3}{*}{$\begin{array}{l}\text { Valor } \\
\text { de p }\end{array}$} \\
\hline & \multicolumn{3}{|c|}{ Positivo } & \multicolumn{3}{|c|}{ Negativo } & \multicolumn{3}{|c|}{ Pai positivo } & \multicolumn{3}{|c|}{ Mãe positiva } & & \\
\hline & $\mathbf{n}$ & $\%^{\mathrm{a}}$ & $\%^{\mathrm{b}}$ & $\mathbf{n}$ & $\%^{a}$ & $\%^{\mathrm{b}}$ & $\mathbf{n}$ & $\%^{a}$ & $\%^{\mathrm{b}}$ & $\mathbf{n}$ & $\%^{\mathrm{a}}$ & $\%^{\mathrm{b}}$ & & \\
\hline \multirow[t]{3}{*}{$\mathrm{Al}$} & $\mathrm{CP}(\mathrm{n}=110)$ & 52 & 47,3 & 60,5 & 17 & 15,5 & 39,5 & 12 & 10,9 & 44,4 & 29 & 26,4 & 55,8 & 0,111 \\
\hline & $\mathrm{CN}(\mathrm{n}=98)$ & 34 & 34,7 & 39,5 & 26 & 26,5 & 60,5 & 15 & 15,3 & 55,6 & 23 & 23,5 & 44,2 & \\
\hline & Todos & 86 & - & 100 & 43 & - & 100 & 27 & - & 100 & 52 & - & 100 & \\
\hline
\end{tabular}

Legenda: $\mathrm{n}(\%)^{\mathrm{a}}(\%)^{\mathrm{b}} ; \mathrm{n}: \mathrm{n}^{\mathrm{o}}$ de adolescentes; ${ }^{\mathrm{a}}$ percentual relativo ao total de alunos; ${ }^{\mathrm{b}}$ percentual relativo ao total de pais; PEV-N: perfil do estilo de vida componente nutrição; $\mathrm{Al}$ : alunos; $\mathrm{CP}$ comportamento positivo dos escolares; $\mathrm{CN}$ comportamento negativo dos escolares.

Tabela 3. Análise da relação do componente atividade física do PEV entre pais e filhos.

\begin{tabular}{|c|c|c|c|c|c|c|c|c|c|c|c|c|c|c|}
\hline \multirow{3}{*}{$\begin{array}{c}\text { PEV- } \\
\text { AF }\end{array}$} & \multicolumn{6}{|c|}{$\begin{array}{l}\text { Casal Concorda } \\
\text { (comportamento) }\end{array}$} & \multicolumn{7}{|c|}{$\begin{array}{c}\text { Casal Discorda } \\
\text { (comportamento) }\end{array}$} & \multirow{3}{*}{ Valor de $\mathrm{p}$} \\
\hline & \multicolumn{3}{|c|}{ Positivo } & \multicolumn{3}{|c|}{ Negativo } & \multicolumn{3}{|c|}{ Pai positivo } & \multicolumn{3}{|c|}{ Mãe positiva } & & \\
\hline & $\mathbf{n}$ & $\%^{a}$ & $\%^{\mathrm{b}}$ & $\mathbf{n}$ & $\%^{\mathrm{a}}$ & $\%^{\mathrm{b}}$ & $\mathbf{N}$ & $\%^{a}$ & $\%^{\mathrm{b}}$ & $\mathbf{n}$ & $\%^{a}$ & $\%^{\mathrm{b}}$ & & \\
\hline \multirow[t]{3}{*}{ Al } & $\mathrm{CP}(n=173)$ & 43 & 24,9 & 95,6 & 49 & 28,3 & 73,1 & 56 & 32,4 & 82,4 & 25 & 14,5 & 89,3 & \multirow{3}{*}{$0,015^{*}$} \\
\hline & $\mathrm{CN}(\mathrm{n}=35)$ & 2 & 5,7 & 4,4 & 18 & 51,4 & 26,9 & 12 & 34,3 & 17,6 & 3 & 8,6 & 10,7 & \\
\hline & Todos & 45 & - & 100 & 67 & - & 100 & 68 & - & 100 & 28 & - & 100 & \\
\hline
\end{tabular}

Legenda: $\mathrm{n}(\%)^{\mathrm{a}}(\%)^{\mathrm{b}} ; \mathrm{n}$ : $\mathrm{n}^{\mathrm{o}}$ de adolescentes; ${ }^{\mathrm{a}}$ percentual relativo ao total de alunos; ${ }^{\mathrm{b}}$ percentual relativo ao total de pais; PEV-AF: perfil do estilo de vida componente atividade física; $\mathrm{Al}$ : alunos; $\mathrm{CP}$ comportamento positivo dos escolares; $\mathrm{CN}$ comportamento negativo dos escolares. 
Tabela 4. Análise da relação do componente comportamento preventivo do PEV entre pais e filhos

\begin{tabular}{|c|c|c|c|c|c|c|c|c|c|c|c|c|c|c|}
\hline \multirow{3}{*}{$\begin{array}{l}\text { PEV- } \\
\text { CP }\end{array}$} & \multicolumn{6}{|c|}{$\begin{array}{c}\text { Casal Concorda } \\
\text { (comportamento) }\end{array}$} & \multicolumn{7}{|c|}{$\begin{array}{c}\text { Casal Discorda } \\
\text { (comportamento) }\end{array}$} & \multirow{3}{*}{$\begin{array}{l}\text { Valor } \\
\text { de p }\end{array}$} \\
\hline & \multicolumn{3}{|c|}{ Positivo } & \multicolumn{3}{|c|}{ Negativo } & \multicolumn{3}{|c|}{ Pai positivo } & \multicolumn{3}{|c|}{ Mãe positiva } & & \\
\hline & $\mathbf{n}$ & $\%^{a}$ & $\%^{\mathrm{b}}$ & $\mathbf{n}$ & $\%^{\mathrm{a}}$ & $\%^{\mathrm{b}}$ & $\mathbf{n}$ & $\%^{\mathrm{a}}$ & $\%^{\mathrm{b}}$ & $\mathbf{n}$ & $\%^{a}$ & $\%^{\mathrm{b}}$ & & \\
\hline \multirow[t]{3}{*}{$\mathrm{Al}$} & $\mathrm{CP}(n=206)$ & 190 & 92,2 & 99 & 1 & 0,5 & 100 & 7 & 3,4 & 100 & 8 & 3,9 & 100 & 0,983 \\
\hline & $\mathrm{CN}(\mathrm{n}=2)$ & 2 & 100 & 1 & - & - & - & - & - & - & - & - & - & \\
\hline & Todos & 192 & - & 100 & 1 & - & 100 & 7 & - & 100 & 8 & - & 100 & \\
\hline
\end{tabular}

Legenda: $\mathrm{n}(\%)^{\mathrm{a}}(\%)^{\mathrm{b}}$; $\mathrm{n}$ : $\mathrm{n}^{\mathrm{o}}$ de adolescentes; ${ }^{\mathrm{a}}$ percentual relativo ao total de alunos; ${ }^{\mathrm{b}}$ percentual relativo ao total de pais; PEV$\mathrm{CP}$ : perfil do estilo de vida componente comportamento preventivo; Al: alunos; $\mathrm{CP}$ comportamento positivo dos escolares; $\mathrm{CN}$ comportamento negativo dos escolares.

Tabela 5. Análise da relação do componente relacionamento do PEV entre pais e filhos

\begin{tabular}{|c|c|c|c|c|c|c|c|c|c|c|c|c|c|c|}
\hline \multirow{3}{*}{ PEV-R } & \multicolumn{6}{|c|}{$\begin{array}{l}\text { Casal Concorda } \\
\text { (comportamento) }\end{array}$} & \multicolumn{7}{|c|}{$\begin{array}{c}\text { Casal Discorda } \\
\text { (comportamento) }\end{array}$} & \multirow{3}{*}{$\begin{array}{l}\text { Valor } \\
\text { de p }\end{array}$} \\
\hline & \multicolumn{3}{|c|}{ Positivo } & \multicolumn{3}{|c|}{ Negativo } & \multicolumn{3}{|c|}{ Pai positivo } & \multicolumn{3}{|c|}{ Mãe positiva } & & \\
\hline & $\mathbf{n}$ & $\%^{a}$ & $\%^{\mathrm{b}}$ & $\mathbf{n}$ & $\%^{a}$ & $\%^{\mathrm{b}}$ & $\mathbf{N}$ & $\%^{a}$ & $\%^{b}$ & $\mathbf{n}$ & $\%^{\mathrm{a}}$ & $\%^{\mathrm{b}}$ & & \\
\hline \multirow[t]{3}{*}{ Al } & $\mathrm{CP}(n=191)$ & 129 & 67,5 & 92,1 & 16 & 8,4 & 94,1 & 19 & 9,9 & 90,5 & 27 & 14,1 & 90 & 0,956 \\
\hline & $\mathrm{CN}(\mathrm{n}=17)$ & 11 & 64,7 & 7,9 & 1 & 5,9 & 5,9 & 2 & 11,8 & 9,5 & 3 & 17,6 & 10 & \\
\hline & Todos & 140 & - & 100 & 17 & - & 100 & 21 & - & 100 & 30 & - & 100 & \\
\hline
\end{tabular}

Legenda: $\mathrm{n}(\%)^{\mathrm{a}}(\%)^{\mathrm{b}} ; \mathrm{n}$ : $\mathrm{n}^{\mathrm{o}}$ de adolescentes; ${ }^{\mathrm{a}}$ percentual relativo ao total de alunos; ${ }^{\mathrm{b}}$ percentual relativo ao total de pais; PEV-R: perfil do estilo de vida componente relacionamentos; $\mathrm{Al}$ : alunos; $\mathrm{CP}$ comportamento positivo dos escolares; $\mathrm{CN}$ comportamento negativo dos escolares.

Tabela 6. Análise da relação do componente controle de estresse do PEV entre pais e filhos.

\begin{tabular}{|c|c|c|c|c|c|c|c|c|c|c|c|c|c|c|}
\hline \multirow{3}{*}{$\begin{array}{l}\text { PEV- } \\
\text { CE }\end{array}$} & \multicolumn{6}{|c|}{$\begin{array}{c}\text { Casal Concorda } \\
\text { (comportamento) }\end{array}$} & \multicolumn{7}{|c|}{$\begin{array}{c}\text { Casal Discorda } \\
\text { (comportamento) }\end{array}$} & \multirow{3}{*}{$\begin{array}{l}\text { Valor } \\
\text { de p }\end{array}$} \\
\hline & \multicolumn{3}{|c|}{ Positivo } & \multicolumn{3}{|c|}{ Negativo } & \multicolumn{3}{|c|}{ Pai positivo } & \multicolumn{3}{|c|}{ Mãe positiva } & & \\
\hline & $\mathbf{n}$ & $\%^{\mathrm{a}}$ & $\%^{b}$ & $\mathbf{n}$ & $\%^{a}$ & $\%^{b}$ & $\mathbf{N}$ & $\%^{a}$ & $\%^{\mathrm{b}}$ & $\mathbf{n}$ & $\%^{\mathrm{a}}$ & $\%^{\mathrm{b}}$ & & \\
\hline \multirow[t]{3}{*}{ Al } & $\mathrm{CP}(n=176)$ & 73 & 41,5 & 84,9 & 25 & 14,2 & 73,5 & 55 & 31,3 & 88,7 & 23 & 13,1 & 88 & 0,230 \\
\hline & $\mathrm{CN}(\mathrm{n}=32)$ & 13 & 40,6 & 15,1 & 9 & 28,1 & 26,5 & 7 & 21,9 & 11,3 & 3 & 9,4 & 11,5 & \\
\hline & Todos & 86 & - & 100 & 34 & - & 100 & 62 & - & 100 & 26 & - & 100 & \\
\hline
\end{tabular}

Legenda: $\mathrm{n}(\%)^{\mathrm{a}}(\%)^{\mathrm{b}} ; \mathrm{n}$ : $\mathrm{n}^{\mathrm{o}}$ de adolescentes; ${ }^{\mathrm{a}}$ percentual relativo ao total de alunos; ${ }^{\mathrm{b}}$ percentual relativo ao total de pais; PEV-CE: perfil do estilo de vida controle de estresse; Al: alunos; $\mathrm{CP}$ comportamento positivo dos escolares; $\mathrm{CN}$ comportamento negativo dos escolares.

tendência dos pais influenciarem positivamente seus filhos nestes componentes do estilo de vida.

Isso pode indicar que o CP de ambos os pais potencializa a possibilidade de terem filhos com $\mathrm{CP}$, mesmo que no caso de ambos os pais ou apenas um deles apresentarem $\mathrm{CN}$ não tenha interferido no CP dos filhos.

\section{Discussão}

Ao se avaliar as relações entre os componentes do PEV de adolescentes e os de seus pais para ajudar em intervenções adequadas, e considerando algumas limitações do estudo, os principais resul- tados apontam que tanto os casais (mães/pais), quanto os filhos possuem um CP para o Estilo de Vida. Ao analisar os resultados dos pais e mães separadamente, verificou-se que as mães foram classificadas com $\mathrm{CN}$ no componente atividade física (1,20 média de pontos) e próximo a um $\mathrm{CN}$ no componente controle de estresse $(1,60$ média de pontos). Estes achados vão ao encontro de estudos que apontam que dentre os adultos as mulheres são menos ativas que os homens ${ }^{13-15}$. As médias da pontuação obtidas pelas mães no controle de estresse aproximaram-se de valores considerados como CN, apoiando resultados de outros estudos que encontraram prevalência maior de estresse em adultos do sexo feminino ${ }^{15,16}$. A 
possível explicação poderia se basear nas atuais exigências sócio-culturais para com as mulheres, de maneira geral, gerando uma sobrecarga de atividades, em que a carreira (profissional ou acadêmica) é acrescida de responsabilidades familiares, sociais e biológicas.

No componente nutrição do PEV observouse que os adolescentes e os pais se aproximaram de um CN ( 1,56 pontos) e no componente atividade física somente os pais (1,58 pontos). Apesar da falta de estudos que utilizam a mesma metodologia para classificar o estilo de vida, o que limita a comparação com outros trabalhos, os resultados da presente pesquisa não corroboram com o estudo realizado com adolescentes por Ferrari et al. ${ }^{17}$ que encontraram uma prevalência baixa de estilo de vida saudável no Município de São Paulo (apenas 15,4\% dos adultos e 9,8\% dos adolescentes) ${ }^{18}$.

Características da escola também têm se mostrado fortemente associados com a QV dos adolescentes ${ }^{19}$. Uma possível explicação para um $\mathrm{CP}$ dos adolescentes do presente estudo se dá por frequentarem um colégio de regimento próprio que é galgado nos valores da disciplina e aonde são valorizadas e ofertadas diversas atividades esportivas extracurriculares gratuitas, assim como a existência de uma carga curricular de educação física obrigatória maior que nas demais redes de ensino (4horas semanais) para esta faixa etária. Isso possibilita aos escolares ter maior contato com um ambiente ativo e motivacional para um comportamento positivo relacionado à saúde, $\mathrm{o}$ qual pode ter refletido no elevado percentual de adolescentes com um PEV positivo, principalmente considerando as associações significativas no componente AF encontrado nos resultados deste estudo.

O fato da média de pontos obtidos nas respostas para o componente nutrição dos adolescentes ter se aproximado de um $\mathrm{CN}$, pode ser reflexo das mudanças nos padrões alimentares ocorridas nos últimos anos, que acabaram contribuindo para a alta incidência de excesso de peso em crianças e adolescentes ${ }^{20,21}$. Entre essas mudanças, destacam-se o aumento do consumo de bebidas açucaradas, alimentos de altas densidades energéticas e pobres em micronutrientes e o baixo consumo de verduras, vegetais e frutas ${ }^{22}$. Já, para os pais, a ocorrência de valores próximos ao negativo pode ser consequência de uma menor preocupação do homem no comportamento alimentar, bem como menor insatisfação com o peso corporal, quando comparado às mulheres. Isso pode ser explicado, pelo fato que os homens têm uma menor auto-percepção corporal do que as mulheres ${ }^{23}$. Raphaelli et al. ${ }^{24}$, ao avaliarem a associação entre os hábitos alimentares dos adolescentes e de seus pais, não encontraram adesão a maioria dos passos da alimentação saudável, recomendados pelo Ministério da Saúde, tanto pelos pais como pelos adolescentes. Isso se torna preocupante, pois recentes estudos indicaram que a alimentação influencia diretamente a saúde das pessoas, tanto positiva como negativamente, e é apontada como um dos fatores mais importantes para a longevidade com $\mathrm{QV}^{25}$.

No presente estudo não foram encontradas relações significativas entre o comportamento do grupo de pais com o do grupo dos filhos nos componentes do PEV, somente no componente atividade física foi encontrada uma relação significativa entre o comportamento do grupo de casais com a do grupo dos filhos $(p=0,015)$. Achados de um estudo realizado por Erkelenz et al. ${ }^{26}$, não demonstraram associação entre os níveis de AF dos pais e das crianças, mas as crianças com pelo menos um dos pais ativo apresentaram maior participação nos esportes organizados sugerindo que o apoio dos pais para AF nas crianças é mais importante do que os pais serem um modelo a seguir. Isso é reforçado em outro estudo que revelou que as barreiras parentais e auto-eficácia estão associados ao tempo que as crianças gastam, tanto na atividade física, quanto em frente à tela. E entre as barreiras investigadas, custos e falta de acesso a instalações foram as mais proeminentes, particularmente a renda e aqueles que vivem em locais rurais ${ }^{27}$.

Apesar disso, os resultados parecem indicar numericamente, tanto nas relações estabelecidas entre grupo de pais (casal) e grupo de filhos para o PEV total como nos seus componentes separadamente, que uma postura conjunta positiva de ambos os pais no casal parece possibilitar o aumento na possibilidade dos filhos terem CP. Vale destacar que no componente nutrição, quando ambos os pais possuem $\mathrm{CN}$ parece crescer a incidência de filhos com $\mathrm{CN}$, e nos outros componentes o $\mathrm{CN}$ de ambos os pais ou a presença em um deles no casal parece não interferir no CP dos filhos. Em um recente estudo de revisão sistemática não se encontrou evidências que crianças de famílias monoparentais tenham especial necessidade de medidas extraordinárias para facilitar seu nível de AF, por exemplo ${ }^{28}$.

Vale destacar que estudos indicam que a percepção que o adolescente tem da qualidade da relação que ela tem com seus pais está positivamente relacionada com a percepção que o ado- 
lescente tem da sua QV, ou seja, se a relação entre o adolescente e os pais for positiva, assim será a sua percepção sobre a sua vida, sendo uma percepção negativa se a relação entre o adolescente e os pais for negativa ${ }^{29}$. Assim, se a relação com os pais for positiva os sintomas psicológicos serão menos frequentes e consequentemente o envolvimento em comportamentos de risco será menor.

\section{Conclusão}

Considerando os aspectos que permearam os dados coletados, verifica-se que, em geral, existe um comportamento positivo prevalente nos componentes do perfil de estilo de vida do grupo estudado. As associações entre o grupo casal e os filhos no componente atividade física demonstraram que um comportamento positivo de ambos os pais pode ter um impacto positivo na vida dos filhos.

Ainda assim, é importante salientar que nos componentes atividade física e controle de estresse das mães e no componente nutrição dos filhos e pais o comportamento verificado foi próximo ao negativo. A elaboração de medidas de saúde no contexto escolar e familiar, de forma conjunta, pode ser uma maneira eficiente de se buscar reduzir a exposição a fatores de risco nos adolescentes, assim promovendo a saúde de toda a família. Atividades promovidas pela escola para os pais em horários alternativos, como atividade físicas festivas, jogos de integração com os filhos no ambiente escolar, palestras, reuniões, podem ser ferramentas salutares para a promoção da saúde de toda a família, bem como uma maneira educativa de aproximar os pais da escola, tornando-os mais proativos na educação da saúde dos filhos.

A falta de estudos e padronização de uma mesma metodologia para classificar o estilo de vida limita a comparação com outros trabalhos. Isso foi considerado como um fator limitante do presente estudo, o que pode ter contribuído para que algumas relações não fossem estatisticamente significativas.

Os resultados do presente estudo podem contribuir no embasamento cientifico para realização de intervenções e planejamento de estratégias de prevenção em médio e longo prazo, a fim de promover mudanças de hábitos e comportamentos ligados ao estilo de vida de toda a família.

\section{Colaboradores}

T Minuzzi realizou toda elaboração do artigo, que teve a contribuição das autoras KCM Pandolfo e CR Azambuja na digitalização e discussão dos resultados. No que se refere no delineamento da metodologia, conclusão e correção do artigo teve auxílio da autora e orientadora de DL Santos. 


\section{Referências}

1. Camargo APPM, Barros Filho AA, Antonio MARDG, Giglio JS. A não percepção da obesidade pode ser um obstáculo no papel das mães de cuidar de seus filhos. Cien Saude Colet 2013; 18(2):323-333.

2. Brito L, Martins R, Cat M, Boguszewski M. Influência da educação em saúde da família no comportamento de risco em adolescentes. Arq Ciênc da Saúde 2016; 23(2):60-64.

3. Wu YP, Reiter-Purtill J, Zeller MH. The role of social support for promoting quality of life among persistently obese adolescents: importance of support in schools. J Sch Health 2014; 84(2):99-105.

4. World Health Organization (WHO). Health for the world's adolescents: a second chance in the second decade. Geneva: WHO; 2014. [acessado $2016 \mathrm{dez} 21$ ]. Disponível em: http://apps.who.int/adolescent/seconddecade/files/1612_MNCAH_HWA_Executive_ Summary.pdf

5. Assumpção Júnior FB, Kuczynski E. Qualidade de vida na infância e na adolescência: orientações para pediatras e profissionais da saúde mental. Porto Alegre: Artmed; 2010.

6. Farias Júnior JC, Nahas MV, Barros MVG, Loch MR, Oliveira ESA, De Bem MFL, Lopes AS. Comportamentos de risco à saúde em adolescentes no Sul do Brasil: prevalência e fatores associados. Rev Panam Salud Pública 2009; 25(4):344-352.

7. Malik VS, Willett WC, Hu FB. Global obesity: trends, risk factors and policy implications. Nature Rev Endocrinology 2013; 9(1):13-27.

8. Nahas MV. Atividade física, saúde e qualidade de vida: conceitos e sugestões para um estilo de vida ativo. $6^{\mathrm{a}}$ ed. Londrina: Midiograf; 2013.

9. Gonçalves A, Vilarta R, organizadores. Atividade Física e Qualidade de Vida: explorando teorias e práticas. Barueri: Manole; 2004.

10. Carvalho CP, Mineiro A. Promoção da saúde e dos estilos de vida saudáveis no contexto escolar português: uma proposta de intervenção lúdica. Democratizar (Faetec) 2012; 6(2). [acessado 2016 Dez 20]. Disponível em: http://www.faetec.ri.gov.br/desup/ images/democratizar/v6-n2/democratizar-20122cristiana-mineiro.pdf

11. Duarte MEB. Influência dos estilos de vida familiar no desenvolvimento do excesso de peso e obesidade em crianças em idade pré-escolar [tese]. Lisboa: Universidade de Lisboa; 2007.

12. Nahas MV, Barros MVG, Francalacci V. O pentáculo do bem-estar: base conceitual para avaliação do estilo de vida de indivíduos ou grupos. Rev Bra Ativ Fís e Saúde 2000; 5(2):48-59.

13. Freire RS, Lélis FLDO, Fonseca Filho JAD. Prática regular de atividade física: estudo de base populacional no Norte de Minas Gerais, Brasil. Rev Bras Med Esp 2014; 20(5):345-349.

14. Mielke GI, Hallal PC, Rodrigues GBA, Szwarcwald CL, Santos FV, Malta DC. Prática de atividade física e hábito de assistir à televisão entre adultos no Brasil: Pesquisa Nacional de Saúde 2013. Epidemiol Serv Saúde 2015; 24(2):277-286.

15. Torquato JA, Goulart AG, Vicentin P, Correa U. Avaliação do estresse em estudantes universitários. Inter Science Place 2010; 1(4):140-154.
16. Paulino CA, Prezotto AO, Frias AC, Bataglia PR, Aprile MR. Sintomas de estresse e tontura em estudantes de pós-graduação. Rev Equilibrio Corporal e Saúde 2010; 2(1):15-26.

17. Ferrari TK, Cesar CLG, Alves MCGP, Barros MBDA, Goldbaum M, Fisberg RM. Healthy lifestyle in São Paulo, Brazil. Cad Saude Publica 2017; 33(1):e00188015.

18. Braz M, Barros Filho AA, Barros M. "Saúde dos adolescentes: um estudo de base populacional em Campinas, São Paulo, Brasil." Cad Saude Publica (2013); 29(9):1877-1888.

19. Barriuso-Lapresa L, Hernando-Arizaleta L, Rajmil L. Social inequalities in mental health and health-related quality of life in children in Spain. Pediatrics 2012; 130(3):528-535.

20. Bento GG, Silva FC, Gonçalves E, Santos PD, Silva R. Revisão sistemática sobre nível de atividade física e estado nutricional de crianças brasileiras. Rev Saude Publica 2016; 18(4):630-642.

21. Souza ADM, Pereira RA, Yokoo EM, Levy RB, Sichieri R. Alimentos mais consumidos no Brasil: Inquérito nacional de alimentação 2008-2009. Rev Saude Publica 2013; 47(1):190-199.

22. Carvalho EADA, Simão MTJ, Fonseca MC, Andrade RGD, Ferreira, MSG, Silva AF, Souza IPR, Fernandes BS. Obesidade: aspectos epidemiológicos e prevenção. Rev Med Minas Gerais 2013; 23(1):74-82.

23. Mascarenhas LPG, Modesto MJ, Amer NM, Boguszewski MCS, Lacerda Filho L, Prati FS. Influência do excesso de peso dos pais em relação ao sobrepeso e obesidade dos filhos. Pensar a Prática 2013; 16(2):519532.

24. Raphaelli CDO, Azevedo MR, Hallal PC. Associação entre comportamentos de risco à saúde de pais e adolescentes em escolares de zona rural de um município do Sul do Brasil. Cad Saude Publica 2011; 27(12):2429-2440.

25. Silva JG, Teixeira MLO, Ferreira MA. Alimentação e saúde: sentidos atribuídos por adolescentes. Esc Anna Nery Rev Enferm 2012; 16(1):88-95.

26. Erkelenz N, Kobel S, Kettner S, Drenowatz C, Steinacker JM, The Research Group "Join the Healthy Boat - Primary School.”. Parental Activity as Influence on Children's BMI Percentiles and Physical Activity. J Sports Sci Med 2014; 13(3):645-650.

27. Smith BJ, Grunseit A, Hardy LL, King L, Wolfenden L, Milat A. Parental influences on child physical activity and screen viewing time: a population based study. BMC Public Health 2010; 10:593.

28. Singhammer J, Ried-Larsen M, Moller NC, Lund-Kristensen P, Froberg K, Andersen LB. Single parent status and children's objectively measured level of physical activity. Sports Med Open 2015; 1(1):1-11.

29. Saha R, Huebner ES, Suldo SM, Valois RF. A longitudinal study of adolescent life satisfaction and parenting. Child Ind Res 2010; 3(2):149-165.

Artigo apresentado em 21/05/2017

Aprovado em 07/02/2018

Versão final apresentada em 09/02/2018 